\title{
A Study to Evaluate Midline Fracture Resistance of Maxillary Acrylic Denture Base Reinforced with Metal Pattern in Various Palatal Contours
}

\author{
Krishna Prasad D ${ }^{1}$, Easha Singh Dogra ${ }^{2}$, Chethan Hegde ${ }^{3}$
}

\begin{abstract}
Aim and objective: The aim and objective of this study was to investigate the effect of reinforcing metal patterns on the midline fracture resistance of maxillary complete denture bases in various palatal contours.

Materials and methods: Sixty maxillary acrylic heat cure denture bases were made. They were subdivided into three groups depending on the palatal vault shape. Further, the groups were subdivided based on the metal pattern design. Three metal pattern designs are used in this study, and the metal patterns are made from 23-gauge stainless steel round wire. The maxillary wax patterns were processed, and metal patterns were reinforced in the packing stage. The denture bases were finished and polished. The samples were checked for inclusion and exclusion criteria. The samples were tested under universal testing machine. The value at which the denture bases fractured were noted down.

Results: Variance analysis test has been done to evaluate the significance. Tukey post hoc test was done to evaluate pairwise analysis of metal patterns and different palatal vault on each other. A significant difference ( $p$ value $<0.05)$ was seen in the fracture resistance value of control group and the reinforced metal group denture base. Among the design of the metal pattern, square-shaped pattern had a significant $(p$ value $<0.05)$ difference in all the palatal vault shape.

Conclusion: The reinforced denture bases with metal patterns had a significant increase in fracture resistance values of the denture bases in all the three palatal vault shapes. Therefore, metal patterns can be used to improve the fracture resistance.

Clinical significance: Metal patterns can be used in clinical cases where shallow and deep palatal vaults is present to reinforce the complete denture prosthesis. By this method reinforcement can be done at a low cost without effecting the esthetics of the dentures.

Keywords: Denture base, Metal pattern, Palatal vault, Reinforcement.

World Journal of Dentistry (2020): 10.5005/jp-journals-10015-1745
\end{abstract}

\section{INTRODUCTION}

Complete loss of the dentition is a universal phenomenon, and this state is referred to as edentulism. One of the treatment options for edentulous patient is complete denture prosthesis. It is one of the most accepted and traditional treatment choices. It is most suitable treatment options for patients having congenital, acquired, anatomic, and economic limitations.

A complete denture prosthesis is a removable prosthesis that replaces the entire dentition, adjacent anatomic structures, and helps conserve the integrity and function of the stomatognathic system. The material used to fabricate complete denture prosthesis should possess adequate mechanical and physical properties as well as have good esthetics and must be nontoxic. From eras to come, complete denture prosthesis has been made either from polymer or from metal. Polymer is the most preferred and routinely used material for fabrication. Many studies are being carried out to alter the structure of polymer or to reinforce the polymer material so as to enhance its structural properties. ${ }^{1,2}$

Denture base made from pure metal or alloys are more structurally durable, have less distortion, have better strength, and are subjected to less fractures of the denture bases under cyclic or static load. The fit of the prosthesis made from metal is more accurate and has better detail reproduction. It is not porous and is a better conductor of heat which helps the patient in appreciating the taste better. The disadvantages are denture bases are heavy, expensive, fabrication is tedious, and are not esthetic.
${ }^{1-3}$ Department of Prosthodontics and Crown and Bridge, AB Shetty Memorial Institute of Dental Sciences, NITTE (Deemed to be University), Mangaluru, Karnataka, India

Corresponding Author: Easha Singh Dogra, Department of Prosthodontics and Crown and Bridge, AB Shetty Memorial Institute of Dental Sciences, NITTE (Deemed to be University), Mangaluru, Karnataka, India, Phone: +91 9041280838, e-mail: eashadogra@gmail. com

How to cite this article: Prasad DK, Dogra ES, Hegde C. A Study to Evaluate Midline Fracture Resistance of Maxillary Acrylic Denture Base Reinforced with Metal Pattern in Various Palatal Contours. World J Dent 2020;11(4):287-293.

Source of support: Nil

Conflict of interest: None

Heat cure acrylic resin is an alternative material that is used to make complete denture prosthesis. It has its own inadequacies with regard to the material properties; however, amalgamation of its properties makes it the most frequently used material for complete denture fabrication. The foremost benefit of this material is its excellent esthetics. Its color can be altered by using internal or external pigments to make it look like patients own gingiva. Other pros for this material are less fabrication cost, easy to fabricate, and has an excellent polished finish on the surface.

Fracture of complete denture takes place mainly because of two reasons, first being flexural fatigue and the other impact force. ${ }^{3}$ 
Flexural fatigue fracture is a time reliant process that happens over a prolonged period of time due to recurring flexing of the denture. The denture develops a crack after continuous flexing under a small load, which ultimately over a period of time leads to fracture. Microcracks can be seen in this type of fracture in the area of stress concentration.

Due to continuous flexing of the material, these microcracks fuse to form bigger cracks that ultimately weaken the material. Fracture of the denture base occurs from the final loading of the denture. Impact failure usually occurs due to a sudden blow to the denture or accidental falling of the denture.

The etiological factor that can lead to the fracture of denture base is difficult to determine. Some of them are stress during chewing, processing errors, and handling of the patient. It may also occur due to porosity in the structure, presence of any crack, or lack of proper adaptation to the residual alveolar ridge.

Different palatal contours affect the retention stability and support of the denture base. Palatal contours are classified as deep palate(V-shaped), medium palate(U-shaped), and shallow palate(flat). It is seen that fracture resistance of denture base also depends on the palatal contour. Flat palate is less prone to fracture resistance. ${ }^{4}$ A study done by Johnson et al. in which the examined 123 palatal shapes in American population, and they observed that $93 \%$ of the edentulous patients had medium-shaped palate, whereas in 4\% patients shallow palate and only in 3\% deep palate was observed in American population. ${ }^{5}$

To improve the fracture resistance of the denture base material, two approaches are followed: either we can improve the strength of the material being used for making denture bases by reinforcing the denture base material or we can reduce the amount of tension being produced in the midline.

Hence, this study was undertaken to assess whether incorporating a metal pattern into the denture base made from conventional heat cure acrylic material can increase the fracture resistance of the denture base. And if at all it is possible, mechanical properties can also be improved with better esthetic properties in conventional heat cure acrylic material.

\section{Materials and Methods}

In this in vitro study, 60 maxillary denture bases were made. They were divided into three subgroups depending on the palatal vault contour.

- Group I deep palatal vault (>12 mm)

- Group II medium palatal vault (6-12 mm)

- Group III shallow palatal vault (<6 mm).

Three casts of the above-mentioned depth were selected (Fig. 1). They were duplicated using irreversible hydrocolloid (DENTSPLY Zelgan 2002 alginate), and denture bases were fabricated on them 20 each group. The thickness of the wax
(Hindustan modelling wax, Hyderabad) pattern fabricated was standardized to $2.5 \mathrm{~mm}$.

Each group had three further subdivisions which where subgrouped as

- Subgroup A with zigzag metal pattern

- Subgroup B with rectangular metal pattern

- Subgroup C with semicircular metal pattern.

All the metal patterns were made with 23-gauge stainless steel wire (Fig. 2). The metal patterns were incorporated at the time of packing the denture bases. Certain standardizations were considered, such as the width of the metal pattern should be $6 \mathrm{~mm}$ for all the patterns. The patterns should be placed 5-7 $\mathrm{mm}$ behind the incisive papilla and 5-7 $\mathrm{mm}$ in front of the posterior palatal seal. Metal patterns of each type was made to reinforce the denture base.

The wax pattern was then flasked in the base of the flask with type Il gypsum product (White gold, India) (Fig. 3). Separating media was applied to the plaster. After the separating media had dried, counterflasking was done with type Il gypsum product (White gold, India). The flask was clamped and kept aside for 20 minutes. Then, the flask and clamp were placed in a water bath having boiling water for dewaxing. After 5 minutes, the wax was removed. The cast and the counter pour were cleaned properly so that there is no residue of modeling wax present on the mold.

When the flask was warm, cold mold seal was applied to the mold area, cast, and the plaster area, and it was allowed to set and then another layer of seal was applied. When the flask cooled down, we started with the packing procedure. Polymer and monomer of polymethyl methacrylate material (Trevalon, Gurugram) were taken in a ratio of 3:1 by volume. The powder and liquid were mixed. The metal pattern was secured in the mid palatine area with the help of some material in stringy stage and then the mold was packed when the material reached dough stage. The flasked was kept under hydraulic press. Acrylization was done by following the short curing cycle. The flask was kept at $74^{\circ} \mathrm{C}$ for $1 \frac{1}{2}$ hours and then at $100^{\circ} \mathrm{C}$ for 30 minutes in the electrical acrylizer. The flask was removed and kept for bench curing for 8 hours.

The denture base was deflasked. Gross trimming was done. All the denture bases were checked. Any denture base with porosity and shift of pattern from mid palatine region was discarded. Only the denture bases without porosity and without shift of metal pattern were kept. The thickness of the denture base was measured using a digital measuring gauge (InSize, Maharashtra). Then, fine trimming and polishing of the denture base were done, and the denture was stored in a box having distilled water in it. Sixty denture bases were fabricated using the same technique.

Then, the denture bases were tested for the value of fracture resistance $(N)$ under the universal testing machine (Krystal equipment, Maharashtra) (Fig. 4). Denture base was kept facing the intaglio surface upward facing the round fixture of the universal testing machine. The force is applied in the center of mid-palatine
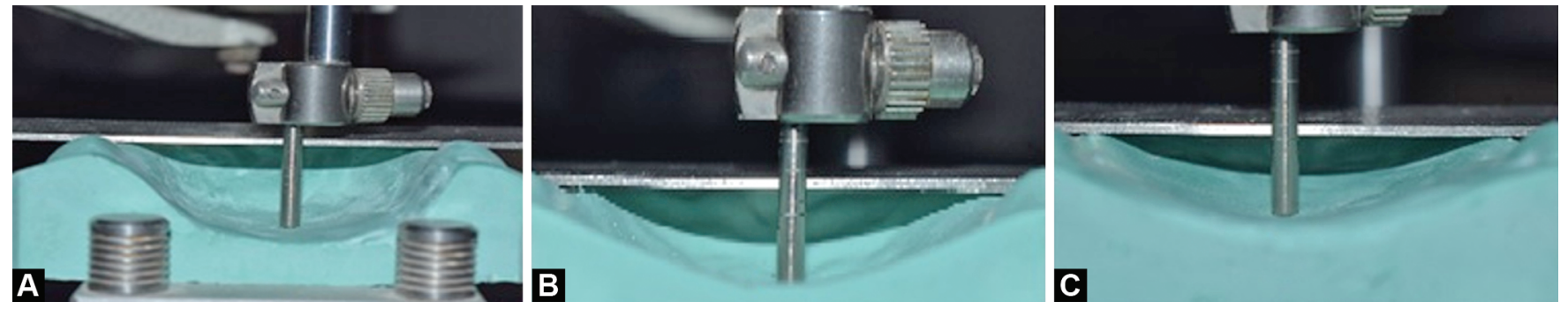

Figs 1 A to C: (A) Deep palatal vault; (B) Medium palatal vault; (C) Shallow palatal vault 



Figs 2A to C: (A) Zigzag metal pattern; (B) Square metal pattern; (C) Semicircular metal pattern

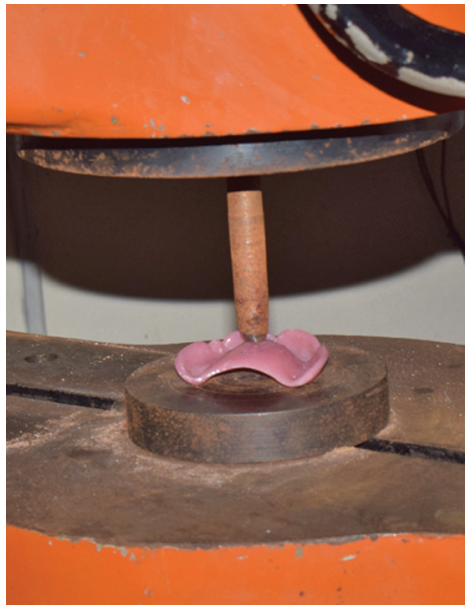

Fig. 4: Testing of the sample under universal testing machine

area corresponding to the second premolar and the molar area. All the 60 denture bases were tested, and the results were noted.

The collected data were entered into Microsoft Excel Spreadsheet and was analyzed using SPSS software (version 20). SPSS statistics interpreted data were presented in the form of mean and standard deviation. ANOVA test was used to compare the mean fracture resistance values between the groups and similarly multiple comparisons between groups were made using post hoc Tukey test. A $p$ value $<0.05$ was considered as statistically significant.

\section{Results}

A total of 60 maxillary heat cure denture bases were made. They were divided into three groups based on the shape of palatal vault. One group had 20 samples each; further the groups where divided into four subgroups based on different design of reinforcing metal pattern. The four subgroups were control group(non-reinforced), zigzag metal pattern, square metal pattern, semicircular metal pattern. Statistical analysis was done to assess the significance of different palatal shape and metal patterns on the fracture resistance of maxillary denture base material. Variance analysis test was done to evaluate the significance of metal patterns and different palatal

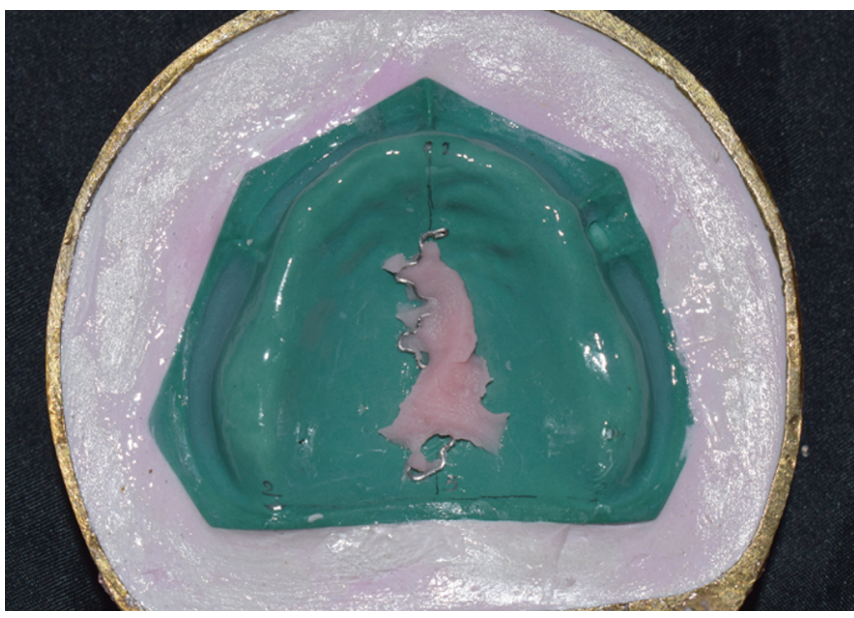

Fig. 3: Packing of the metal pattern

vault shape on fracture resistance ( $p$ value $<0.05$ ). Tukey post hoc test was done to evaluate pairwise analysis of metal patterns and different palatal vault on each other.

When comparing reinforced groups and the control group in each palatal vault shape, it is observed that a significant difference $(p$ value $<0.05$ ) is present between the control group denture bases and the reinforced denture bases in all the palatal vault groups (Fig. 5). It can be observed that the minimum value of fracture resistances of all the reinforcing groups is higher than the maximum value of all the control groups (Table 1). Comparison between all the metal patterns is done in each palatal vault group. It is seen that square metal pattern has a significant increase in fracture resistance when compared to other metal patterns in each group of palatal vault shape (Table 2).

When comparing fracture resistance between metal patterns, it is observed that medium palatal contour has the highest fracture resistance when compared to deep and shallow in the control group (Table 3). Zigzag and square pattern showed a significant difference ( $p$ value $<0.05$ ), but the values obtain with semicircular pattern were nonsignificant ( $p$ value $>0.05$ ) in all the palatal contours.

In the control group, significant difference $(p$ value $<0.05)$ is seen in medium, deep, and shallow palatal vault, and medium palatal samples have the highest fracture resistance values. Zigzag metal pattern had a significant difference $(p$ value $<0.05)$ in medium palatal vault, whereas no significant difference was seen between shallow palate and deep palate. Square metal pattern had a similar effect when compared to the zigzag pattern on the different palatal vaults. Semicircle metal pattern did not have much significant difference $(p$ value $>0.05$ ) (Table 4).

\section{Discussion}

Conventional complete denture prosthesis is a mainstay treatment option available for the rehabilitation of edentulous patient. The prosthesis not only satisfies patients esthetics and functional demands but also provides a very economical treatment option. The material of choice for its fabrication depends on the patient's preference, anatomy, and functional need. The available options for fabrication are polymethylmethacrylate or metal.

Most accepted material for fabrication is polymethylmethacrylate. The positives of the material being good finish, nontoxic effect, low fabrication cost; however, a major drawback is its mechanical 


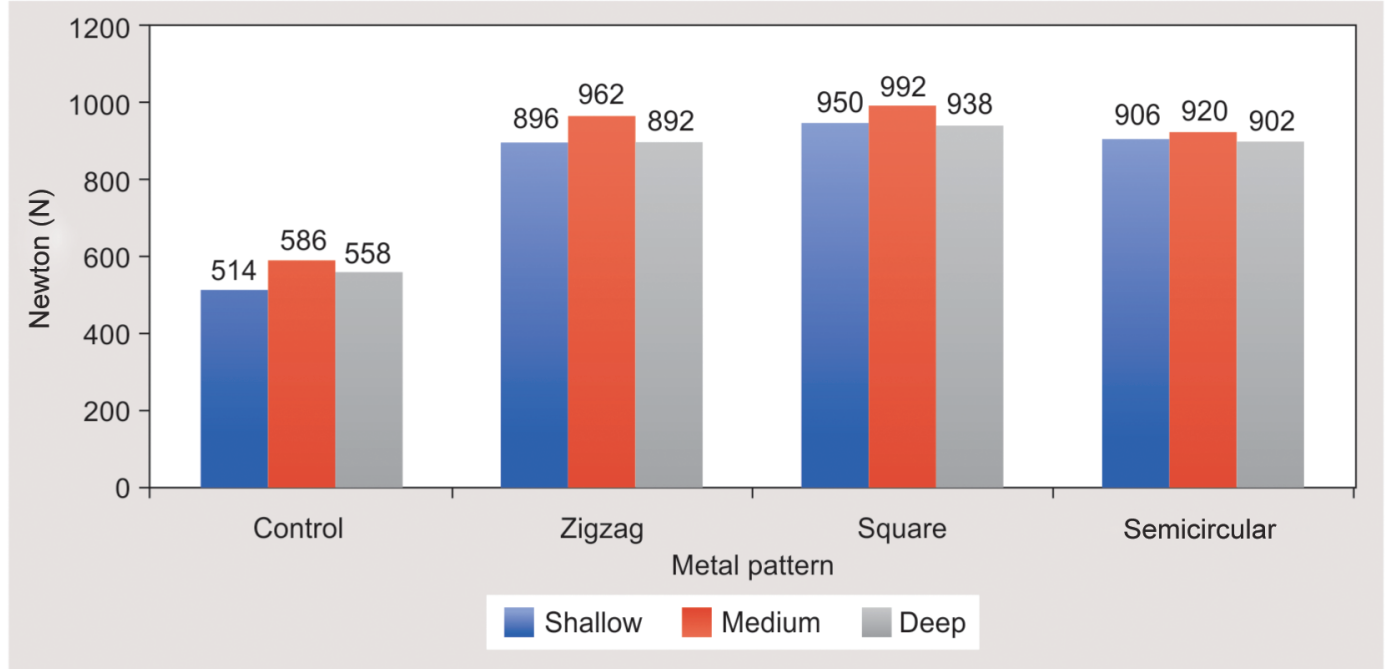

Fig. 5: Fracture resistance in each palatal vault shape

Table 1: Comparison of fracture resistance between different metal pattern in each palate type

\begin{tabular}{|c|c|c|c|c|c|c|c|c|}
\hline \multirow[b]{2}{*}{ Palate type } & \multirow[b]{2}{*}{ Metal pattern } & \multirow[b]{2}{*}{$N$} & \multirow[b]{2}{*}{ Mean } & \multirow[b]{2}{*}{ Std. deviation } & \multirow[b]{2}{*}{ Minimum } & \multirow[b]{2}{*}{ Maximum } & \multicolumn{2}{|c|}{ ANOVA } \\
\hline & & & & & & & $F$ & $p$ value \\
\hline \multirow[t]{4}{*}{ Shallow } & Control & 5 & 514 & 16.73 & 500 & 540 & 1114.05 & $<0.001^{\mathrm{a}}$ \\
\hline & Zigzag & 5 & 896 & 11.40 & 880 & 910 & & \\
\hline & Square & 5 & 950 & 10 & 940 & 960 & & \\
\hline & Semicircular & 5 & 906 & 15.17 & 890 & 920 & & \\
\hline \multirow[t]{4}{*}{ Medium } & Control & 5 & 586 & 11.40 & 570 & 600 & 1244.49 & $<0.001^{\mathrm{a}}$ \\
\hline & Zigzag & 5 & 962 & 13.04 & 950 & 980 & & \\
\hline & Square & 5 & 992 & 8.37 & 980 & 1000 & & \\
\hline & Semicircular & 5 & 920 & 14.14 & 910 & 940 & & \\
\hline \multirow[t]{4}{*}{ Deep } & Control & 5 & 558 & 13.04 & 540 & 570 & 1657.04 & $<0.001^{\mathrm{a}}$ \\
\hline & Zigzag & 5 & 892 & 8.37 & 880 & 900 & & \\
\hline & Square & 5 & 938 & 8.37 & 930 & 950 & & \\
\hline & Semicircular & 5 & 902 & 8.37 & 890 & 910 & & \\
\hline
\end{tabular}

${ }^{\mathrm{a}} p<0.05$ statistically significant; $p>0.05$ nonsignificant (NS)

characteristics. The material is prone to fractures, both flexural fractures and impact fractures. Many alterations in the material have been done to increase the mechanical properties. Metal denture bases have better mechanical properties when compared to heatcured dentures, but their fabrication cost is high, and esthetics are not that pleasing to the patients.

Reports of fracture of maxillary dentures is two times more common when compared to mandibular dentures. Most common etiology for fracture is cyclic masticatory forces and impact force applied on the denture. Midline fractures are the most common as a prominent midline acts as a fulcrum around which the forces are concentrated which further leads to fracture suggested by Schneider et al. ${ }^{6}$ Usually seen in patients who have severe alveolar ridge resorption as the upward forces increases in two halves of the denture around the midline which may lead to fatigue failure around midline of maxillary complete denture.

To increase the fracture resistance of the complete denture prosthesis, many methods have been tried. Some of the methods are chemical alterations in the denture base material, additions of metal wires and meshes, or incorporation of glass fibers, carbon fibers, nylon fibers, vegetable fibers, etc. All the studies have shown significant difference in mechanical properties of complete denture prosthesis material with various reinforcing procedures. ${ }^{7-10}$

Although the literature explains plethora of techniques for reinforcing acrylic denture base material, there are few studies assessing and comparing the effect of reinforcing different metal patterns in various palatal vault contours. Hence the present study was undertaken to evaluate and compare the fracture resistance of maxillary denture base.

In the present study, maxillary denture bases of three different palatal contours, having control, and reinforced with three different metal patterns were fabricated. A total of 60 denture bases where fabricated. All the samples were tested for fracture resistance value using universal testing machine. The load was applied on the intaglio surface in the midline premolar and molar area so as to stimulate the forces present in the oral cavity. This is because most fracture occurs in the midline, and this is in agreement with Morris et al.'s ${ }^{11}$ studies. A round tip fixture was used, and load was 
Table 2: Pairwise comparison of fracture resistance between different metal pattern in each palate type

\begin{tabular}{|c|c|c|c|c|c|c|c|}
\hline \multirow[b]{2}{*}{ Palate type } & \multirow[b]{2}{*}{ (I) Metal pattern } & \multirow[b]{2}{*}{ (J) Metal pattern } & \multirow[b]{2}{*}{ Mean difference $(I-J)$} & \multirow[b]{2}{*}{ Std. error } & \multirow[b]{2}{*}{$p$ value } & \multicolumn{2}{|c|}{ 95\% confidence interval } \\
\hline & & & & & & Lower bound & Upper bound \\
\hline \multirow[t]{6}{*}{ Shallow } & Control & Zigzag & -382 & 8.60 & $<0.001^{\mathrm{a}}$ & -406.61 & -357.39 \\
\hline & & Square & -436 & 8.60 & $<0.001^{\mathrm{a}}$ & -460.61 & -411.39 \\
\hline & & Semicircular & -392 & 8.60 & $<0.001^{\mathrm{a}}$ & -416.61 & -367.39 \\
\hline & Zigzag & Square & -54 & 8.60 & $<0.001^{\mathrm{a}}$ & -78.61 & -29.39 \\
\hline & & Semicircular & -10 & 8.60 & 0.66 (NS) & -34.61 & 14.61 \\
\hline & Square & Semicircular & 44 & 8.60 & $0.001^{\mathrm{a}}$ & 19.39 & 68.61 \\
\hline \multirow[t]{6}{*}{ Medium } & Control & Zigzag & -376 & 7.55 & $<0.001^{\mathrm{a}}$ & -397.6 & -354.4 \\
\hline & & Square & -406 & 7.55 & $<0.001^{\mathrm{a}}$ & -427.6 & -384.4 \\
\hline & & Semicircular & -334 & 7.55 & $<0.001^{\mathrm{a}}$ & -355.6 & -312.4 \\
\hline & Zigzag & Square & -30 & 7.55 & $0.005^{\mathrm{a}}$ & -51.6 & -8.4 \\
\hline & & Semicircular & 42 & 7.55 & $<0.001^{\mathrm{a}}$ & 20.4 & 63.6 \\
\hline & Square & Semicircular & 72 & 7.55 & $<0.001^{\mathrm{a}}$ & 50.4 & 93.6 \\
\hline \multirow[t]{6}{*}{ Deep } & Control & Zigzag & -334 & 6.16 & $<0.001^{\mathrm{a}}$ & -351.64 & -316.36 \\
\hline & & Square & -380 & 6.16 & $<0.001^{*}$ & -397.64 & -362.36 \\
\hline & & Semcircular & -344 & 6.16 & $<0.001^{\mathrm{a}}$ & -361.64 & -326.36 \\
\hline & Zigzag & Square & -46 & 6.16 & $<0.001^{\mathrm{a}}$ & -63.64 & -28.36 \\
\hline & & Semicircular & -10 & 6.16 & 0.40 (NS) & -27.64 & 7.64 \\
\hline & Square & Semicircular & 36 & 6.16 & $<0.001^{\mathrm{a}}$ & 18.36 & 53.64 \\
\hline
\end{tabular}

Tukey post hoc test

${ }^{a} p<0.05$ statistically significant; $p>0.05$ nonsignificant (NS)

Table 3: Comparison of fracture resistance between different palate type in each metal pattern

\begin{tabular}{|c|c|c|c|c|c|c|c|c|}
\hline \multirow[b]{2}{*}{ Metal pattern } & \multirow[b]{2}{*}{ Palate type } & \multirow[b]{2}{*}{$N$} & \multirow[b]{2}{*}{ Mean } & \multirow[b]{2}{*}{ Std. deviation } & \multirow[b]{2}{*}{ Minimum } & \multirow[b]{2}{*}{ Maximum } & \multicolumn{2}{|c|}{ ANOVA } \\
\hline & & & & & & & $F$ & $p$ value \\
\hline \multirow[t]{3}{*}{ Control } & Shallow & 5 & 514 & 16.73 & 500 & 540 & 34.07 & $<0.001^{\mathrm{a}}$ \\
\hline & Medium & 5 & 586 & 11.40 & 570 & 600 & & \\
\hline & Deep & 5 & 558 & 13.04 & 540 & 570 & & \\
\hline \multirow[t]{3}{*}{ Zigzag } & Shallow & 5 & 896 & 11.40 & 880 & 910 & 62.65 & $<0.001^{\mathrm{a}}$ \\
\hline & Medium & 5 & 962 & 13.04 & 950 & 980 & & \\
\hline & Deep & 5 & 892 & 8.37 & 880 & 900 & & \\
\hline \multirow[t]{3}{*}{ Square } & Shallow & 5 & 950 & 10 & 940 & 960 & 50.25 & $<0.001^{\mathrm{a}}$ \\
\hline & Medium & 5 & 992 & 8.37 & 980 & 1000 & & \\
\hline & Deep & 5 & 938 & 8.37 & 930 & 950 & & \\
\hline \multirow[t]{3}{*}{ Semicircular } & Shallow & 5 & 906 & 15.17 & 890 & 920 & 2.68 & 0.11 (NS) \\
\hline & Medium & 5 & 920 & 14.14 & 910 & 940 & & \\
\hline & Deep & 5 & 902 & 8.37 & 890 & 910 & & \\
\hline
\end{tabular}

${ }^{a} p<0.05$ statistically significant; $p>0.05$ nonsignificant (NS)

applied at $10 \mathrm{~mm} /$ minute, the reading at which the denture base fractured was noted. Results were tabulated and fracture resistance was assessed within the three palatal contours, three metal patterns and the control group.

Comparing the results of control group obtained from the present study, it is evident that the load required to fracture the medium vault denture bases is more when compared the shallow vault and the difference between the two is significant $p$ value $<0.05)$. This may be due to the reason of increase in surface area in medium vault. It is suggested in the literature as surface area increases the force per $\mathrm{mm}$ load decreases which leads to better resistance of fracture. ${ }^{12}$ This result is similar to a study done by
Sowmya et al., ${ }^{12}$ where they are comparing two heat cure material in different palatal vault. Similar results were also seen in studies done by Morris et al. ${ }^{11}$

Considering the values of control group in deep palatal vault, the group required more load as compared to the shallow palate and less load from medium palate to fracture. All the values had a significant difference $(p$ value $<0.05)$. The result obtained in the current study is in accordance with the study done by Reddy et al. ${ }^{4}$ and is not in correlation with previous studies done by Morris et al. who concluded in his study with 45 sample size that there is no significant difference between deep palate and medium palate. The findings in the present study are different because there was 
Table 4: Pairwise comparison of fracture resistance between different palate type in each metal pattern

\begin{tabular}{|c|c|c|c|c|c|c|c|}
\hline \multirow[b]{2}{*}{ Metal pattern } & \multirow[b]{2}{*}{ (I) Palate type } & \multirow[b]{2}{*}{ (J) Palate type } & \multirow[b]{2}{*}{ Mean difference (I-J) } & \multirow[b]{2}{*}{ Std. error } & \multirow[b]{2}{*}{ pvalue } & \multicolumn{2}{|c|}{ 95\% confidence interval } \\
\hline & & & & & & Lower bound & Upper bound \\
\hline \multirow[t]{3}{*}{ Control } & Shallow & Medium & -72.00 & 8.79 & $<0.001^{\mathrm{a}}$ & -95.46 & -48.54 \\
\hline & & Deep & -44.00 & 8.79 & $0.001^{a}$ & -67.46 & -20.54 \\
\hline & Medium & Deep & 28.00 & 8.79 & $0.02^{\mathrm{a}}$ & 4.54 & 51.46 \\
\hline \multirow[t]{3}{*}{ Zigzag } & Shallow & Medium & -66.00 & 7.02 & $<0.001^{\mathrm{a}}$ & -84.74 & -47.26 \\
\hline & & Deep & 4.00 & 7.02 & 0.84 (NS) & -14.74 & 22.74 \\
\hline & Medium & Deep & 70.00 & 7.02 & $<0.001^{\mathrm{a}}$ & 51.26 & 88.74 \\
\hline \multirow[t]{3}{*}{ Square } & Shallow & Medium & -42.00 & 5.66 & $<0.001^{\mathrm{a}}$ & -57.09 & -26.91 \\
\hline & & Deep & 12.00 & 5.66 & 0.13 (NS) & -3.09 & 27.09 \\
\hline & Medium & Deep & 54.00 & 5.66 & $<0.001^{\mathrm{a}}$ & 38.91 & 69.09 \\
\hline \multirow[t]{3}{*}{ Semicircular } & Shallow & Medium & -14.00 & 8.17 & 0.24 (NS) & -35.78 & 7.78 \\
\hline & & Deep & 4.00 & 8.17 & 0.88 (NS) & -17.78 & 25.78 \\
\hline & Medium & Deep & 18.00 & 8.17 & 0.11 (NS) & -3.78 & 39.78 \\
\hline
\end{tabular}

Tukey post hoc test

${ }^{\mathrm{a}} p<0.05$ statistically significant; $p>0.05$ nonsignificant (NS)

a standardization of the denture base thickness and the depth of palatal vault.

Akyil et al. ${ }^{13}$ conducted a study in which they reinforced denture bases made in different palatal vault shapes with round stainless-steel wire, half round stainless-steel wire, and metal mesh. They observed that all the reinforced samples had greater fracture resistance as compared to the non-reinforced samples.

In the present study, the data that are obtained show a significant difference between the control groups and all the experimental groups, thus suggesting that reinforcing the denture bases with metal pattern helped in enhancing the fracture resistance of the denture bases. This may be due to the fact that metal pattern allowed for more deflection of the acrylic resin.

The location of the reinforcing material also helps in increasing the fracture resistance. There are two methods for that either reinforcing the entire denture base material or reinforcing only that area where stress accumulation in expected more. In a study done by Komala et al., ${ }^{14}$ reinforcement was done using complete and partial glass fiber mesh. A significant difference was seen between the two groups from the control group. In the present study. partial reinforcement has been done at the sight most prone to fractures.

Three metal designs were fabricated with stainless-steel wire to reinforce the mid palatine region of the maxillary denture base. The designs made were zigzag pattern, Square pattern, and semicircular pattern. It was observed that all the metal patterns had a significant increase in the mean reinforcement values from the control group in each palatal vault groups. One of the observations was that square metal pattern had the greatest mean difference among all the metal pattern in all the palatal vault groups. This may be due to the design of the metal pattern. Square-shaped metal pattern had more perpendicular components in its design when compared to the other patterns. It has been suggested by Ruffino et al. ${ }^{15}$ that when two metal wires are placed a few $\mathrm{mm}$ apart at $90^{\circ}$ to the probable line of fracture, a significant increase is seen in the reinforcing ability of the denture base.

The results of the present study provide a new insight into the relationship between fracture resistance and palatal contour. In general, $93 \%$ of the population has medium palatal vault, $4 \%$ have shallow palatal vault and $3 \%$ deep palatal vault the dentist can evaluate and reinforce the denture if he feels is required. The metal patterns that are being used in the present study have never been used previously in the literature and is an easy way of increasing the fracture resistance of the denture base without any deteriorating effect on the esthetic aspect of the denture and is a very cost-effective reinforcing technique.

\section{Conclusion}

Within the limitation of the study the following conclusion were drawn:

- From the result of the study, it was found out that denture bases reinforced with metal patterns had increased fracture resistance when compared to the non-reinforced denture bases.

- Medium palatal vault had increased fracture resistance in all the groups thus concluding that palatal shape influences the fracture resistance

- Among the metal pattern square shaped metal pattern had highest fracture resistance values whe compared to other metal patterns

\section{Clinical Significance}

Metal patterns can be used in clinical cases where shallow and deep palatal vaults is present to reinforce the complete denture prosthesis. It can be used in patients having neuromuscular disorders to prevent fracture of complete dentures due to impact force. By this method reinforcement can be done at a low cost without effecting the esthetics of the dentures.

\section{References}

1. Jagger $D C$, Harrison A, Jandt KD. The reinforcement of dentures. J Oral Rehabil 1999;26(3):185-194. DOI: 10.1046/j.1365-2842.1999.00375.x.

2. Im SM, Huh YH, Cho LR, et al. Comparison of the fracture resistances of glass fiber mesh-and metal mesh-reinforced maxillary complete denture under dynamic fatigue loading. J Adv Prosthodont 2017;9(1):22-30. DOI: 10.4047/jap.2017.9.1.22.

3. Smith DC. Recent developments and prospects in dental polymers. J Prosthet Dent 1962;12(6):1066-1078. DOI: 10.1016/00223913(62)90162-2. 
4. Reddy BM, Himabindu M, Padmaja BI, et al. Palatal vault depth influence on the flexural strength of two heat cure acrylic denture base resins: an in vitro study. J Contemp Dent Pract 2013;14(6):1131. DOI: $10.5005 /$ jp-journals-10024-1463.

5. Johnson DL, Holt RA, Duncanson JrMG. Contours of the edentulous palate. J Am Dent Assoc 1986;113(1):35-40. DOI: 10.14219/jada. archive.1986.0147.

6. Schneider RL. Diagnosing functional complete denture fractures. J Prosthet Dent 1985;54(6):809-814.DOI: 10.1016/0022-3913(85)90476-7.

7. Murthy HM, Shaik S, Sachdeva H, et al. Effect of reinforcement using stainless steel mesh, glass fibers, and polyethylene on the impact strength of heat cure denture base resin-an in vitro study. J Inter Oral Health: JIOH 2015;7(6):71.

8. Pan Y, Liu F, Xu D, et al. Novel acrylic resin denture base with enhanced mechanical properties by the incorporation of PMMA-modified hydroxyapatite. Prog Nat Sci: Mat Inter 2013;23(1):89-93. DOI: 10.1016/j.pnsc.2013.01.016.

9. Gad MM, Fouda SM, Al-Harbi FA, et al. PMMA denture base material enhancement: a review of fiber, filler, and nanofiller addition. Int J Nanomedic 2017;12:3801. DOI: 10.2147/IJN.S130722.
10. Tacir IH, Kama JD, Zortuk M, et al. Flexural properties of glass fibre reinforced acrylic resin polymers. Aust Dent J 2006;51(1):52-56. DOI: 10.1111/j.1834-7819.2006.tb00401.x.

11. Morris JC, Khan Z, von Fraunhofer JA. Palatal shape and the flexural strength of maxillary denture bases. J Prosthe Dent 1985;53(5): 670-673. DOI: 10.1016/0022-3913(85)90018-6.

12. Sowmya S, Dhakshaini MR, Gujjari AK, et al. Palatal vault depth influence on the flexural strength of two heat cure acrylic denture base resins - an in vitro comparative study. World Appl Sci J 2013;21(9):1290-1294.

13. Duymuş ZY, Akyil MŞ, Denizoğlu S. An investigation of fracture loads of acrylic base plates. Atatürk Üniversitesi Diş Hekimliği Fakültesi Dergisi 2006;2006(1):18-24.

14. Jayasree Komala DP, Bheri S. To evaluate the fracture resistance of maxillary complete dentures reinforced with full and partial glass fibre mesh: an in vitro study. Int J Med Health Res 2018;4(8):181-187. DOI: 10.22271/ijmhr.2018.v4.i8.36.

15. Anthony R. Ruffino. effect of steel strengtheners on fracture resistance of the acrylic resin complete denture base. J Prosthe Dent 1985;54(1):75-78. DOI: 10.1016/S0022-3913(85)80074-3. 